\title{
Aortoesophageal Fistula Caused by Descending Aortic Pseudoaneurysm
}

\author{
S MAHMOOD $^{\mathrm{a}}$, MM HOSSAIN ${ }^{\mathrm{b}}$, MA HOSSAIN $^{\mathrm{c}}$
}

\begin{abstract}
Summary:
A 60 year old man presented in Square Hospitals LTD with hypovolaemic shock, and massive haematemesis. His BP was non-recordable with soft, non-tender abdomen and $\mathrm{Hb}$ was $8 \mathrm{~g} / \mathrm{dl}$ with normal prothrombin time and partial thromboplastin time and normal platelets.By endoscopy and CT scans aortoesophageal fistula with aortic pseudoaneurysm
\end{abstract}

\section{Introduction:}

Aortoesophageal fistula (AOF) is a rare cause of gastrointestinal haemorrhage. Early diagnosis and surgical intervention are mandatory for survival. We report a case of an aorto-esophageal fistula due to a descending aortic pseudoaneurysm and review the causes, clinical manifestations, and diagnosis of this uncommon but often fatal case of upper gastrointestinal haemorrhage.

Computed tomography is the most valuable diagnostic method in the diagnosis of aortoesophageal fistula.CT may detect the aneurysm, its relation to the oesophagus and surrounding structures.It can also differentiate between true and false aneurysms. Once AOF is identified, immediate surgery is mandatory.

\section{Case report:}

The patient, a 60 year old man, presented with hypovolumic shock and massive haematemesis. The patient had repeated episodes of massive haematemesis \& malaena without dysphagia, chest pain or loss of weight. The patient was detected HbsAg. positive incidentally. He was a smoker. On admission, his blood pressure was non-recordable and pulse rate was 125 beats/min, confirming haemorrhagic shock. Physical examination included a soft, non-tender abdomen with normal bowel sounds. Additional results were

a. Dr Shagufta Mahmood, Clinical Fellow ( NUH, Singapore), Consultant, Radiology and Imaging, Square Hospitals Ltd.

b. Dr M. Motahar Hossain, Consultant, Hepatology \& Gastroenterology, Square Hospitals Ltd.

c. Dr. M. Akhter Hossain, Consultant, Cardiac surgery, Square Hospitals Ltd.

Address of Correspondence : Dr Shagufta Mahmood, Apt : A-4, House 72, Road 11/A, Dhanmondi R/A, Dhaka 1209, Email: sa.azhar@yahoo.com

Received: 2 March 2015

Accepted: 24 Nov. 2016 was identified and immediate surgery was done with complete occlusion of the pseudoaneurysm, which was confirmed by repeat CT scan.

Key wards: Aortoesophageal fistula, Aortic pseudo aneurysm.

(J Bangladesh Coll Phys Surg 2017; 35: 34-35)

haemoglobin $8 \mathrm{~g} / \mathrm{dl}$, creatinine $0.9 \mathrm{mg} / \mathrm{dl}$, normal prothrombin and partial thromboplastin times and normal platelets. Chest X-ray was normal.

After receiving and 5 units of blood, the patient was stabilized and after that endoscopy was performed. Under endoscopy, a large globular mass compressing

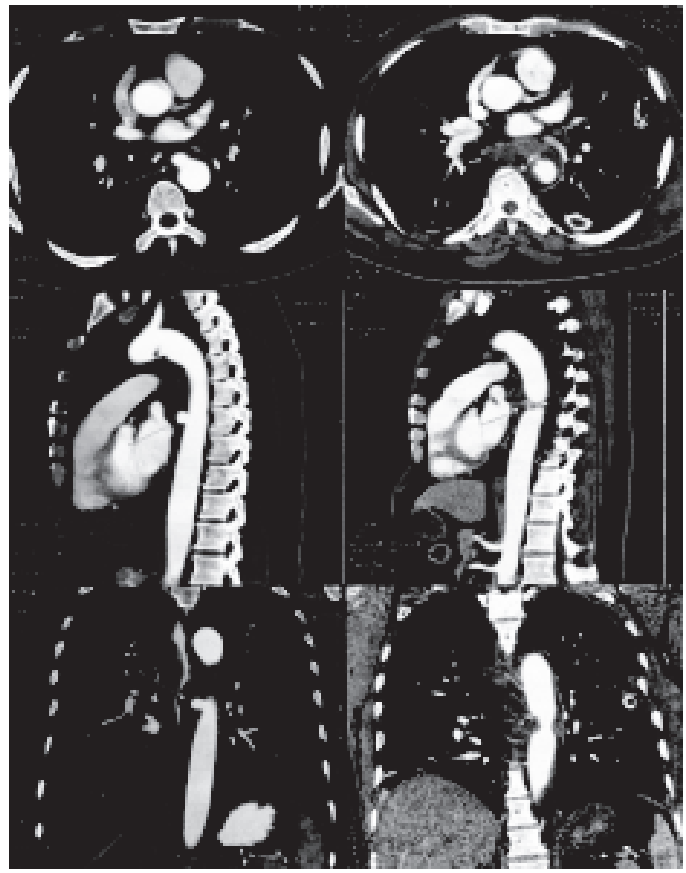

Fig.-1

the mid oesophagus was seen at $30 \mathrm{~cm}$ from the incisor teeth. A visible blood vessel was seen at the base of the ulcer at the tip of the mass. The vessel started spurting of the blood during the endoscopic process. Haemostasis was achieved instantly by injecting $10 \mathrm{ml}$ of $5 \%$. Ethanolaminoleate was injected in and around the bleeding vessels. 
A subsequent contrast enhanced computed tomographic scan of the chest documented an outpouching at the antero-medial aspect of the mid part of descending aorta measuring approx. $2.5 \mathrm{~cm} \times 1.3 \mathrm{~cm}$ in size suggesting oesophageal pseudoaneurysm ( Fig.1). The aneurysm is situated approx. $6 \mathrm{~cm}$ distal to the isthmus, compressing and indenting the adjacent oesophageal lumen. Oesophageal wall at this site was mildly thickened. This may suggest aorto-esophageal fistula. Two small $9 \mathrm{~mm}$ right paraoesophageal lymphnodes are also detected.

Urgent surgery was planned to excise or occlude the pseudoaneurysm. Descending thoracic aorta was approached by left posterolateral thoracotomy through $4^{\text {th }}$ intercostal space. Aneurysmal sac was identified and found badly adherent to the surrounding soft tissue. Decision was made to occlude the base of pseudoaneurysm and to avoid excessive difficult dissection. Control of aorta was taken both proximal and distal to the pseudoaneurysm. Purse string suture was given at the base of sac by 3-0 prolene. Blood pressure was lowered to $70 \mathrm{~mm} \mathrm{Hg}$ and purse string suture tied. Two additional transfixation suture of 3-0 prolene through the aneurysmal sac were given and tied down. Haemostasis was achieved and chest was closed by keeping a drain tube.

A subsequent CT confirmed that the pseudoaneurysm was completely occluded (Fig. 2). The patient recovered well before discharge.

\section{Discussion :}

Development of a fistula between the aorta and oesophagus has an extremely poor prognosis. In 1991 Hollander and Quick (1) published a comprehensive review, including 500 cases of AEF gathered from the literature. The main aetiological factor involved aortic disease with $54.2 \%$ of cases being secondary to rupture of an aneurysm of the descending thoracic aorta into the esophagus. The next most frequent causes were foreign body (19.2\%) and advanced oesophageal cancer $(17.0 \%)$. Secondary AEF following operative treatment were rare (4.8\%) with 50\% occurring after aortic surgery and $50 \%$ after oesophageal surgery. Diagnosis of AEF is rarely made before massive haematemesis. However most cases are associated with characteristic CHIARI's triad of aorto oesophageal syndrome, including chest pain and sentinel haematemesis of red blood followed at a variable interval of time by rapidly fatal massive exsanguinating haematemesis. Chracterestic triad features were present in $45 \%$ of patients included in the review series of Hallander' Quick (2), 80\% of patients describe by Carter et $\mathrm{al}^{2,3}$. In a few cases AEF can be suspected on the basis of isolated sepsis or septic embolism in a lower extremity. Our suggestive findings include dysphagia and or chest pain, H/O surgical treatment of thoracic aorta.

Except in patients who require immediate emergency surgery, various investigative modalities can be used to confirm diagnosis, plan treatment.

Barium oesophagus may show compression by the aneurysm of the oesophagus ${ }^{4}$.

Esophagoscopy can also show the presence of a variable amount of sub-mucosal haematoma indicating extravasation of blood into the oesophageal wall ${ }^{5}$. However even though some investigations advocate oesophagoscopy as the diagnostic modality of choice for evaluation of AEF, others have advised against its use because several cases of fatal haemorrhage precipitated by flexible endoscopy have been reported ${ }^{6}$.

Although arteriogram rarely reveal the fistula site they can provide useful data for surgical planning.

\section{Conclusion:}

Pre operative contrast enhanced CT chest of the patient revealed the aorto-oesophageal fistula with pseudoaneurysm and post operative CT demonstrated complete occlusion of the tract (Fig1).So, CT scan is a valuable diagnostic method for both pre operative diagnosis and post operative follow-up in these patients.

\section{Acknowledgement:}

We remain indebted to Director, Square Hospitals Ltd. for his permission to publish this report.

\section{References:}

1. Hollander JE,Quick. G. Aorto esophageal fistula : A comprehensive review of the literature.Am J Med - 1991; $91: 279-287$

2. Yasuda F, Shimono T, Tonouchi H, et al : Successful repair of an aorto oesophageal fistula with aneurysm from oesophageal diverticulum. Ann Thorac.Surgery -2002;73 : 637-639.

3. Center R, Mulder GA, Snyder EN Jr, et al : Aorto oesophageal fistula. Am J surg. 1978;136: 26-30

4. Xia Min, GVO Ji-zhong, ZHAN Qiang,et al. Aortooesophageal fistula caused by descending aortic pseudoaneurysm : one case report . Chinese medical journal 2007;120 ( 23):2149-2150

5. Myers HS, Silber W. oesophageal bleeding form aortooesophageal fistula due to aortic aneurysm : case reports and review of literature, S Afr med. $1983 ; 63 ; 124-127$

6. Baron RL,KoehlerRE, Gutierrez FR, et al . clinical and radiological manifestation of aorto oesophageal fistulas . Radiology . 1981; 141: 599-605. 